\title{
Protein structure and function analysis for plant stress tolerance on BTB/POZ genes
}

\author{
Anashkina A.A. ${ }^{1}$, Luzin A.N. ${ }^{2}$, Kulikova D.K. ${ }^{2}$, Dergilev A.I. ${ }^{2}$, Orlov Y.L. ${ }^{2,3,4 *}$ \\ ${ }^{1}$ Engelhardt Institute of Molecular Biology RAS, Moscow, Russia \\ ${ }^{2}$ Novosibirsk State University, Novosibirsk, Russia \\ ${ }^{3}$ Peoples' Friendship University of Russia (RUDN University), Moscow, Russia \\ ${ }^{4}$ Institute of Cytology and Genetics, SB RAS, Novosibirsk, Russia \\ *email: orlov@bionet.nsc.ru
}

The DNA sequencing has allowed us to determine the full genomes of many plants, including model organisms such as Arabidopsis thaliana, scientifically interesting species of flowering plants, trees, and economically important crops such as rice, maize, soy, cotton, and wheat. Developments in omics technologies have enabled unprecedented views of plants across scales, from the molecular to the ecosystem level, demanding integration of large, noisy, and heterogeneous data sets. There are several bioinformatics modeling fields in plants: Detailed analysis of regulatory elements of gene expression (transcription factors, regulatory regions, chromatin state, alternative splicing mechanisms); Methods to discern differences among members of a gene family, particularly large homologous gene families, and to identify gene copies likely to be functionally redundant; computational modeling of high-resolution molecular structures of proteins/enzymes and assemblies with to identify and prioritize structural features affecting plant cell response to environment conditions (stress tolerance). The expression analyses showed that the defending type drought stress tolerance contributing LAN C like protein GCL-2 is expressed in providing disease resistance and myb linked $\mathrm{BTB} / \mathrm{POZ}$ genes contribute for high yielding of pigeonpea. BOP is a member of BTB group of plant protein. The BOP genes encode proteins containing a BTB/POX virus and zinc finger (POZ) domain and ankyrin repeat. BLADE-ON-PETIOLE 1 (BOP1) and BOP2 encoding genes are redundant transcription factors restricted to the base of developing lateral organs including the leaf and floral development. Regulatory network inference is a prime example of the usefulness of bioinformatics in plant sciences. Recent examples are the inference of tissue-specific gene regulatory networks in maize, of a dynamic gene regulatory network related to nitrogen use efficiency in Arabidopsis, and of regulatory networks coordinating timing and rate of gene expression in response to environmental signals in rice. Putative encoding region of transit peptides and mature proteins from different plant genomes were predicted using Blast2go. Domain Prediction of lanc-like protein GCL2 and BOP gene in Cajanus was done by NCBI CD-search software. Fasta sequence of BOP genes was retrieved from NCBI followed by Blast search. RaptorX structure prediction tool was used to analyze distantly related template proteins (especially those with sparse sequence profiles) and quality of the alignment. We discussed the structural protein models obtained. Unraveling the effects of genotypic variation on protein structure provide fundamental insights into the regulation of plant development and physiology and the ability to predict yield and quality traits. 